\title{
Determining the Role of Gene Expression Assays in Local Therapy for Breast Cancer: First Steps with a Positive Impression
}

\author{
Mackenzie Daly, MD \\ Department of Radiation Oncology, Washington University School of Medicine, St Louis
}

Determining when post-mastectomy radiation therapy (PMRT) should be utilized in breast cancer patients with $\mathrm{T} 1$ or $\mathrm{T} 2$ tumors and $1-3$ positive lymph nodes is an area of uncertainty. In this patient population, balancing the benefits of improved local control and overall survival with the long-term complications of therapeutic radiation relies heavily on the 'art of medicine'. ${ }^{1,2}$ The paper submitted by Dr. Jegadeesh and colleagues published in this issue of Annals of Surgical Oncology suggests that the 21-gene recurrence score assay may be a tool to optimize local therapy for breast cancer patients. Furthermore, the authors offer a novel perspective on utilizing the recurrence score for clinical decision making. ${ }^{3}$

For the reader, it is helpful to appreciate the precarious position that PMRT has held in treatment paradigms. Early trials, such as the DBCG 82 b\&c trials, included patients with one or more positive axillary nodes and showed improved local control as well as improved survival. ${ }^{4,5}$ Questions regarding the adequacy of surgery and older systemic agents used in these trials make many clinicians wary about applying historic results to modern-day patients.

By the late 1990s a consensus report was released stating that insufficient evidence exists to make evidencebased recommendations regarding PMRT in patients with small tumors and 1-3 positive axillary lymph nodes. ${ }^{6,7}$ More recently, the treatment pendulum has been swinging back with the results of the Early Breast Cancer Trialist's Collaborative Group meta-analysis, reporting reduction of

(C) Society of Surgical Oncology 2014

First Received: 5 November 2014; Published Online: 2 December 2014

M. Daly, MD

e-mail: mdaly@radonc.wustl.edu locoregional recurrence (LRR) and improvement in breast cancer mortality for this patient population. ${ }^{2}$

In the absence of clear, randomized data, oncologists are forced to weigh clinicopathologic risk factors, ultimately relying on their clinical judgment to recommend or not recommend PMRT in patients with small primary tumors (T1-T2) and 1-3 positive lymph nodes. While tallying up an individual patient's risk factors for local recurrence, lymphovascular invasion, tumor size, nodal involvement, extracapsular extension, age, etc., I cannot help but reflect on my medical oncology colleagues. They too were once weighing clinical and pathologic risk factors down to the millimeter, using actuarial science to treat individual patients with biologically heterogeneous tumors. ${ }^{8,9}$ That is until the 21-gene recurrence score became widely available, allowing medical oncologists to tailor systemic therapy recommendations for estrogen receptor (ER)positive, HER2-negative breast cancer patients by integrating their individual molecular profiles. ${ }^{10,11}$

Although systemic management of early-stage breast cancer patients has moved forward into a genomic era, local therapies such as radiation and surgery continue to treat cancer empirically. In the current issue of Annals of Surgical Oncology, the article entitled "21-Gene Recurrence Score and Locoregional Recurrence in Breast Cancer Patients" 3 attempts to clarify if there is a relationship between the 21-gene recurrence score and local recurrence. The authors retrospectively reviewed records of stages I and II, ER-positive breast cancer patients who were treated with breast-conserving therapy (BCT) or mastectomy alone. Patients received systemic therapy based upon their 21-gene recurrence score. The study was limited by rather marked differences in patient and tumor characteristics for each local control group. Most notably, there was a statistically significant difference in patient age, stage, and lymph node status between the BCT and mastectomy-alone groups. Additionally, there was a very low event rate in the 
study, with only 11 LRRs in the entire cohort. As the authors of this paper highlighted, a low local recurrence rate is expected in stages I and II breast cancer patients. Despite these limitations, a relationship was identified between recurrence score and mastectomy-alone patients. An association between recurrence score and LRR has been examined in two prior retrospective analyses. ${ }^{12,13}$

The retrospective analysis of the National Surgical Adjuvant Breast and Bowel Project (NSABP) B-14 and B-20 trials evaluated ER positive, node-negative breast cancer patients enrolled in these two trials. ${ }^{12}$ In NSABP B-14, participants were randomized to 5 years of placebo versus tamoxifen; local therapy could have been either BCT or mastectomy without radiation therapy. ${ }^{14}$ NSABPB20 was a prospective randomized trial with three arms that randomly assigned patients to tamoxifen alone or tamoxifen with one of two systemic therapy regimens. ${ }^{15}$ The authors of the NSABP-14 and NSABP-20 retrospective review concluded that recurrence score had a significant association with the risk of local and regional recurrence. ${ }^{12}$ There were some unusual findings that suggested radiation may be more effective in patients with higher recurrence scores. Jegadeesh and colleagues ${ }^{3}$ took note that in the NSABP B-14 and B-20 review, a high recurrence score predicted for LRR in all mastectomy-alone patients, while recurrence score did not predict for LRR in breast-conservation patients over the age of 50 years. They felt that this further substantiated their hypothesis that radiotherapy may in some way nullify the LRR implications of a high recurrence score. This hypothesis was further supported by a second and more recent retrospective review of the EORTC E2197 trial. ${ }^{13}$ This trial evaluated the recurrence score in ER-positive, node-positive patients, all of whom received BCT. Interestingly, no association between recurrence score and risk of local recurrence was identified.

I would like to commend the authors of this article for utilizing recurrence scores in a less traditional way. Rather than adopting the original systemic therapy stratification system of $\mathrm{RS}<18$, RS $18-30$, and $\mathrm{RS} \geq 31$ as low, intermediate, and high risk, respectively, they correlated locoregional outcomes to recurrence scores to mathematically find the most significant relationship. This is an approach that is reasonable in that the threshold for scores for risk of local recurrence may not mirror thresholds for systemic recurrence.

"The 21-Gene Recurrence Score and Locoregional Recurrence in Breast Cancer Patients" retrospective review provides fodder for future prospective trials, advancing the dialogue regarding local therapy and asking how we can better select treatments for individuals with cancer.

\section{REFERENCES}

1. Clarke M, Collins R, Darby S, et al. Effects of radiotherapy and of differences in the extent of surgery for early breast cancer on local recurrence and 15-year survival: an overview of the randomized trials. Lancet. 2005;366(9503):2087-16.

2. McGale P, Taylor C, Correa C, et al. Effect of radiotherapy after mastectomy and axillary surgery on 10-year recurrence and 20year breast cancer mortality: meta-analysis of individual patient data for 8135 women in 22 randomized trials. Lancet. 2014: 383(9935):2127-35.

3. Jegadeesh N, Kim S, Oprea G, et al. The 21-gene recurrence score and locoregional recurrence in breast cancer patients. Ann Surg Oncol.

4. Overgaard M, Hansen PS, Overgaard J, et al. Postoperative radiotherapy in high-risk premenopausal women with breast cancer who receive adjuvant chemotherapy. Danish Breast Cancer Cooperative Group 82b Trial. N Engl J Med. 1997;337(14): 949-55.

5. Overgaard M, Jensen MB, Overgaard J, et al. Postoperative radiotherapy in high-risk postmenopausal breast-cancer patients given adjuvant tamoxifen. Danish Breast Cancer Cooperative Group DBCG 82c Trial. Lancet. 1999;353(9165):1641-8.

6. Recht A, Edge SB, Solin LJ, et al. Postmastectomy radiotherapy: clinical practice guidelines of the American Society of Clinical Oncology. J Clin Oncol. 2001;19(5):1539-69.

7. Wallgreen A, Bonbetti M, Gelber RD, et al. Risk factors for locoregional recurrence among breast cancer patients: results from International Breast Study Group Trials I through VII. $J$ Clin Oncol. 2003;21(7):1205-13.

8. Cheng S, Horng C, Clarke J, et al. Prognostic index score and clinical prediction model of local regional recurrence after mastectomy in breast cancer patients. Int J Radiat Oncol Biol Phys. 2006;64(5):1401-9.

9. Fisher B, Dignam J, Tan-Chiu E, et al. Prognosis and treatment of patients with breast tumors of one centimeter or less and negative axillary nodes. J Natl Cancer Inst. 2001;93(2):112-20.

10. Paik S, Shak S, Tang G, et al. A multigene assay to predict recurrence of tamoxifen-treated, node-negative breast cancer. $N$ Engl J Med. 2004;351(27):2817-26.

11. Albain K, Barlo W, Shak S, et al. Prognostic and predictive value of the 21-gene recurrence score assay in postmenopausal women with node-positive, oestrogen receptor-positive breast cancer on chemotherapy: a retrospective analysis of a randomized trial. Lancet. 2010;11(1):55-65.

12. Mamounas E, Tang G, Fisher B. Association between the 21-gene recurrence score assay and risk of locoregional recurrence in node-negative, estrogen receptor-positive breast cancer: results from NSABP B-14 and BSABP B-20. J Clin Oncol. 2010;28(10): 1677-83.

13. Solin LJ, Gray R, Goldstein LJ, et al. Prognostic value of biologic subtype and the 21-gene recurrence score relative to local recurrence after breast conservation treatment with radiation for early stage breast carcinoma: results from the Eastern Cooperative Oncology Group E2197 study. Breast Cancer Res Treat. 2012;134(2):683-92.

14. Fisher B, Costantino J, Redmond C, et al. A randomized clinical trial evaluating tamoxifen in the treatment of patients with nodenegative breast cancer who have estrogen-receptor-positive tumors. N Engl J Med. 1989;320(8):479-84.

15. Fisher B, Dignam J, Wolmark N, et al. Tamoxifen and chemotherapy for lymph node-negative, estrogen receptor-positive breast cancer. J Natl Cancer Inst. 1997;89(2):1673-82. 